\title{
Dinámica del consumidor que compra a los vendedores ambulantes de la ciudad de Loja
}

\section{Dynamics of the consumer who buys street vendors from the city of Loja}

\author{
Whinzon Patricio Cuenca ${ }^{1}$, Johvana Aguirre Mendoza $^{1}$ y José Rodrigo Maldonado ${ }^{1}$ \\ ${ }^{1}$ Universidad Nacional de Loja \\ *whinzon.cuenca@unl.edu.ec
}

DOI: https://doi.org/10.26871/killkana_social.v2i3.87

\begin{abstract}
Resumen
Como parte del proyecto de investigación "Eficacia de la regulación municipal de las ventas ambulantes en la ciudad de Loja", estas obedecen a una estructura de oportunidad, se presenta como una alternativa para el emprendimiento empírico en el Ecuador a la incapacidad que tiene la economía formal para generar fuentes de empleo en cantidad y calidad, que permita suplir las necesidades de las familias especialmente de escasos recursos económicos, esta investigación revisa el corolario de la demanda, al analizar los factores que inciden en la decisión y calidad de compra de los consumidores que adquieren los productos, y con ello obtener información que tiende a generar soluciones alternativas a la prohibición que se basa en el ornato y la legalidad de las actividades económicas en la ciudad. No se trata de un estudio de mercado sino de una investigación social que articula las dinámicas sociales y económicas, para ello el uso de instrumentos cualitativos y cuantitativos en un enfoque de investigación mixta, parte de un censo basado en la observación directa y se complementa con una encuesta para su validación. El procesamiento de datos se realizó mediante la utilización de técnicas estadísticas, lo cual permite extraer conclusiones de la dinámica comercial como un juego de actores y no sólo de consumidores. Conocer el comportamiento de actores sociales y consumidores permite visualizar soluciones considerando variables geoespaciales: 1. Procedencia. 2. Movilidad. 3. Variedad de productos. 4. Frecuencia de compra. 5. Motivo de compra. 6. Accesibilidad 7. Atención al cliente 8. Moda. 9. Precio cómodo 10. Sensibilidad. 11. Satisfacción, entre otros.
\end{abstract}

Palabras clave: sensibilidad, variable geoespacial, validación, corolario de la demanda.

\begin{abstract}
As part of the research project "Effectiveness of municipal regulation of street sales in the city of Loja", street sales obey an opportunity structure, is presented as an alternative for empirical entrepreneurship in Ecuador to the disability that has the formal economy to generate sources of employment in quantity and quality, which can meet the needs of families with limited economic resources, presents how this research reviews the corollary of demand (supply), by analyzing the factors that affect the decision and quality of purchase of the consumers that acquire the products, and with this obtain information that tends to generate alternative solutions to the prohibition based on the ornament and the legality. It is not a market study but a social research that articulates the social and economic dynamics, for this the use of qualitative and quantitative instruments in a context of mixed research, part of a census based on observation and complemented by a validation focal group. The process of data through the use of statistical techniques allows conclusions to be drawn from commercial dynamics as a set of actors and not just consumers. Knowing the behavior of social actors and consumers allows us to visualize solutions considering geospatial variables: 1. Origin. 2. Mobility. 3. Variety of products. 4. Frequency of purchase. 5. Purpose of purchase. 6. Accessibility 7. Customer service 8. Fashion. 9. Comfortable price 10. Sensitivity. 11. Satisfaction, among others.
\end{abstract}

Key words: sensitivity, geospatial variable, validation, corollary of the demand.

\section{Introducción}

Las ciudades latinoamericanas en las últimas décadas han vivido una metamorfosis urbana caracterizada por su metropolización expandida y conjuntamente, asociado a las desigualdades socioespaciales, un proceso de fragmentación (De Mattos, 2010). La difusión de las TIC, la intensifi- cación de la conectividad y la movilidad y la liberalización del suelo urbano, han ampliado las opciones de localización de las personas y facilitado el distanciamiento de los sectores residenciales del centro de la ciudad, aumentando la movilidad cotidiana de las personas y los flujos al interior de la metrópolis. 
Las nuevas condiciones como el creciente número de personas instruidas, el aumento de las horas de la fuerza laboral en las oficinas y la nueva tendencia de trabajo a distancia o desde el hogar así como la distribución del ingreso de acuerdo al nivel socioeconómico, el rápido incremento de la clase media y los mercados y las oportunidades provenientes de las nuevas tecnologías (Kotler \& Armstrong, 2008), son variables que definen la actual dinámica que se genera entre compradores y vendedores de ventas ambulantes en la ciudad de Loja.

En este contexto los ciudadanos en sus movilidades cotidianas, requieren satisfacer múltiples necesidades que los convierten en "consumidores ambulantes" (Durán, 2013), demandando prácticas móviles de consumo en sus espacios de tránsito cotidiano, ya sea el transporte público o en la calle, que el comercio establecido satisface sólo parcialmente, por lo que el comercio ambulante emerge como una alternativa para responder a estas demandas, a través de la apropiación del espacio público, como la calle, la micro, la esquina o el semáforo.

De esta manera, el comercio informal se constituye en una estrategia informal de empleo, se convierte en una práctica que permite dar respuesta a las necesidades de quienes realizan movilidades cotidianas, siendo en este sentido los clientes más ambulantes que los propios vendedores (Durán, 2013). Como señala Salazar (2013), la venta ambulante resulta de la relación entre vendedores y un movimiento libre de consumidores, que se despliegan en un flujo permanente

La presente investigación consideró herramientas y técnicas de investigación social como los es el censo a los vendedores ambulantes y la encuesta dirigida a los consumidores que acuden a estos, seleccionándose mediante un muestreo estratificado de acuerdo a la variable localización de los vendedores en las calles, paradas y semáforos de la ciudad de Loja, el periodo de estudio está considerado dentro el proyecto de investigación eficacia de la regulación municipal de las ventas ambulantes en la ciudad de Loja, desde sus orígenes hasta la actual realidad socioeconómica en la que se desarrollan estas actividades consideradas informales de la economía.

Se consideró variables decisorias como condiciones socioeconómicas de los consumidores, productos de expendio de las ventas ambulantes, frecuencia de compra, geolocalización, motivos de compra, reacción rápida a las necesidades del consumidor y percepciones del consumidor para una compra efectiva.

Es importante mencionar que la escasa colaboración de los consumidores para brindar la información clara y precisa, por temas de orden cultural como el miedo, desconfianza y urgencia, fueron factores que limitaron la información, pero se encontró como resultados que existe una diferenciación de estos motivos encontrando que para el canal informal prima las relaciones sociales establecidas, la forma de entrega, disponibilidad del producto y valor agregado del servicio.
Es importante el trabajo investigativo puesto que brinda una alternativa a la solución del problema de ventas ambulantes en la ciudad de Loja, desde un punto de vista del consumidor y sus características psico-socioeconómicas, mismas que no están divorciadas de la objetividad respetando ciertos preceptos metodológicos, constituyéndose de esta forma una propuesta de desarrollo económico sustentable y de planificación local.

\section{Marco teórico}

\subsection{La elección de compra}

Se describe a continuación las variables identificadas en algunos estudios: Spiggle \& Sewall (1987) elaboraron un modelo de elección del punto de venta por parte del consumidor enfocado en tres aspectos: a) los estados sicológicos del consumidor: actitudes, percepciones, imagen, pesos de los atributos b) las características del consumidor: orientación de compra, características demográficas y estilos de vida c) las características del punto de venta: distancia, surtido y tiempo recorrido. Posteriormente, Diez De Castro (1997) expone que la elección de un punto de venta implica la consideración de tres elementos: a) La existencia o no de una preferencia por parte del consumidor b) que el consumidor haya realizado una compra previa c) la fidelidad que se produce cuando el consumidor ha realizado compras de manera reiterada. Por su lado, Vigaray (2005) clasifica en dos categorías el proceso de comportamiento de compra realizado por el consumidor según la complejidad de la decisión de compra: hacer la compra e ir de mercado (Cardona Madeline, 2016)

Existen otras investigaciones mismas que se concentraron en identificar las características individuales de los consumidores, coinciden que los factores relevantes para realizar las compras en un supermercado o hipermercado son la disponibilidad de bienes en el momento que se requieren de acuerdo con (Farhangmehr, Marques y Silva, 2000) mientras que la elección de compra en la tienda tradicional depende de la distancia a recorrer y la tendencia a encontrar productos perecederos como lo sostiene (Goldman y Hino, 2005). Gary y Peter (2011) examinaron las actitudes y creencias de los compradores de productos comestibles en el contexto minorista australiano, encontrando que los factores de elección más importantes para los hombres son la velocidad, comodidad y eficiencia; mientras que para las mujeres son los precios, limpieza y calidad.

\subsection{Consumo}

El consumo según (Canclini, 1995). Es comprendido, ante todo, por su racionalidad económica además estudios de diversas corrientes consideran el consumo como un momento del ciclo de producción y reproducción social, es el lugar en el que se completa el proceso iniciado al generar productos, donde se realiza la expansión del capital y se reproduce la fuerza de trabajo. Desde tal enfoque, no son las necesidades o los gustos individuales los que determinan qué, cómo y quiénes consumen. (pag.49). 
El consumo no es una actividad exclusivamente utilitaria, y al mismo tiempo que está sujeto a restricciones materiales, implica prácticas a menudo idiosincrásicas (Duhau Giglia, 2007)

\subsection{Consumidor Ambulante}

Cuando se reconoce que al consumir también se piensa, se elige y reelabora el sentido social hay que analizar cómo interviene esta área de apropiación de bienes y signos en formas más activas de participación que las que habitualmente se ubican bajo el rótulo de consumo. En otros términos, debemos preguntarnos si al consumir no estamos haciendo algo que sustenta, nutre y hasta cierto punto constituye un nuevo modo de ser ciudadanos (Canclini, 1995, pág. 41)

\subsection{Factores psicológicos}

La calidad de vida en la modernidad puede tener un impacto psicológico y social que acarrea la introducción de determinados hábitos, valores y modos de vida en extremo consumistas que pueden suponer un grave peligro para la salud física, el equilibrio psíquico y el bienestar personal o familiar (Garces, 1999)

\subsection{Marketing y titletamiento del consumidor}

Hablar de la soberanía del consumidor. Probablemente sea algo exagerado, pero la satisfacción de las necesidades impone la orientación al consumidor en la actividad de marketing de la empresa, pues una empresa que trabaje sin enfoque de marketing fabrica productos e intenta venderlos a los posibles consumidores, mientras que una empresa que trabaja con enfoque de marketing fabrica productos que sabe anticipadamente que puede vender porque los necesitan y desean los consumidores (Esteban, 2013)

En este sentido el comportamiento del consumidor es el factor más dinámico, indeterminado, estocástico e impredecible al que ha de hacer frente cualquiera interesado en él. Marketing. En cada decisión comercial y en muchas políticas comerciales subyace una consideración previa relativa al individuo que acude al mercado a realizar sus compras. La dificultad de cada una de estas decisiones comienza con el conocimiento del individuo-consumidor. Sería arriesgado tratar de elaborar estrategias y planes sin una buena dosis de conocimiento de cómo y por qué se forman las preferencias de los consumidores

\subsection{Uso de estitle públicos}

Las ventas callejeras en la medida que contribuyen a la congestión del espacio público, pueden irradiar externalidades negativas al comercio establecido; a la vez, su presencia al reducir la movilidad del público aumenta los costos de tránsito por los espacios congestionados, lo que disminuye las preferencias por estas zonas. Por consiguiente, induce a una menor actividad del comercio formal a través de una reducción de sus clientes potenciales. Así, la congestión del espacio público ocasionada por las ventas callejeras, equivale a una reducción en la oferta de un bien público de uso local o un recurso común, por la rivalidad en su consumo, traduciéndose en una desvalorización de la actividad comercial establecida (Rocha, Sanchez, y Garcìa, 2009, p.248)

\section{Metodología}

La presente investigación se desarrolló en la ciudad de Loja- Ecuador, se definió como objeto de estudio a los compradores de productos de consumo masivo, medicina tradicional, animales menores, comidas y bebidas preparadas listas para servirse, productos para el hogar, prendas de vestir, de uso personal y para el hogar, artículos electrónicos, artículos electrónicos, servicios de recargas, artículos de plástico, cigarrillos y demás bienes o lo que es ventas ambulantes. Se encontró consumidores que presentan características socioeconómicas propias de los barrios adyacentes al centro de la ciudad. La investigación se realizó bajo un enfoque cuantitativo a través del censo a vendedores ambulantes. La interpretación de los datos desde el enfoque cuantitativo permite construir y proyectar un escenario alternativo hacia la construcción de una solución de desarrollo sustentable. Desde lo cualitativo permitió interpretar el sentido y el significado del comportamiento del consumidor objeto de estudio y de los factores internos y externos que lo afectan. Esta observación de las personas investigadas permitió explorar y describir la expresión de los componentes psicológicos, como la sensibilidad, las estrategias de mercadeo y otros factores intrínsecos que conllevan a la decisión de compra de los consumidores.

Para recolectar la información, desde este enfoque, se implementó una encuesta estructurada con cuadros de doble entrada, mismos que se destinaron a 384 consumidores, que es la muestra calculada para poblaciones finitas, que corresponde a la Población en Edad de Trabajar del cantón Loja, según proyecciones poblacionales el INEC al 2018, con un nivel de confianza del $95 \%$, y un nivel de error estándar del $5 \%$, todo esto tomado a partir del muestreo aleatorio simple. Los encuestadores se distribuyeron en sitios estratégicos donde regularmente se sitúan los vendedores ambulantes, cuya estrategia fue que interioricen con los investigados, lo que permitió conocer desde su perspectiva las actitudes de los consumidores y la elección en la compra, en aspectos como sitio de compra y la frecuencia de visita.

Para el análisis de la información, se utilizaron técnicas de análisis multivariado usando el método de Análisis de Componentes Principales (ACP). Es decir, a partir de un número de variables es posible obtener un número reducido de componentes conformados por variables pero con la menor pérdida de información posible. Este método multivariante se aplica a variables numéricas que tienen una relación lineal entre ellas. 


\section{Resultados}

Este estudio se focaliza en la ciudad de Loja, ubicada en el sur del Ecuador, con cerca de 240000 habitantes, según proyecciones del INEC al 2018, en la que se desenvuelven dinámicas entre compradores y vendedores de ventas ambulantes mismas que necesitan ser atendidas de manera urgente.

Los procesos observados en la ciudad, obedecen a tendencias que ocurren con la urbanización de la poblaciones rurales, que buscan ser incluidas en las capitales provinciales, mismas que migran por temas de pobreza, empleo y estudio; con lo cual se resquebraja el tejido económicosocial, por lo que presionados por temas de inserción social y ante la falta de fuentes de empleo en el sector formal de la economía, se toma como alternativa de subsistencia las ventas ambulantes.

Los resultados son presentados en cuatro incisos claves, empezando por las características socioeconómicas del consumidor ambulante; seguido de la frecuencia de compra, movilidad del comprador y tipo de artículos que adquiere; seguidamente se analiza las motivaciones de compra y por último el sitio habitual de compra a los vendedores ambulantes, en zonas previamente delimitadas por los investigadores.

\subsection{Características socioeconómicas de los compradores ambulantes}

Cuando se definió las características socioeconómicas del consumidor ambulante, en los sectores previamente definidos, se encontró que el $76 \%$ de la población de consumidores ambulantes están comprendidos en el rango de edad de 19 a 50 años, donde prevalecen aquellos del rango de edad comprendido entre los 19 a 29 años de edad, con un $35 \%$ de personas y en menores porcentajes el resto de rangos de edad; el sexo de los compradores no tiene ninguna diferencia sustancial; cuando se les indagó sobre el estado civil se encontró que el $83 \%$ son solteros y casados; la ocupación de los compradores son estudiantes, empleados públicos y empelado privados, sumados representan el $69 \%$ de los consumidores de ventas ambulantes; al preguntarles sobre el nivel educativo de los consumidores se observa que $45 \%$ tiene educación superior, y $29 \%$ han estudiado hasta el bachillerato. Es importante mencionar que las parroquias de donde acuden a comprar a los vendedores ambulantes son el Valle y Sucre que conjuntamente representan el $38 \%$ de personas que compran a los vendedores ambulantes, esto porque en estas parroquias se encuentran la mayoría de barrios urbano marginales que toman transporte colectivo para acudir al centro por temas de gestiones o trabajo, y en porcentajes significativos provienen de las parroquias Sagrario, Punzara, San Sebastián y Carigan. Es importante mencionar que existen consumidores de las parroquias rurales del cantón y provincia de Loja y otras provincias que están de paso por la ciudad.
4.2 Tipo de artículos, frecuencia de compra y movilidad del comprador

\subsubsection{Movilidad de los consumidores}

\section{a.- Movilidad hacia su lugar de trabajo}

Cuando los consumidores ambulantes acuden a su sitio de trabajo en forma diaria estos consumen regularmente refrigerios para el trabajo en forma diaria en un $29 \%$ y el $21 \%$ consumen comidas y bebidas preparadas listas para servirse; durante la semana compran refrigerios para llevar al trabajo el $36 \%$ de encuestados, y frutas y verduras el $29 \%$. Compran en fines de semana un $25 \%$, confitería; cigarrillos y licores tiene consumo semanal, fines de semana y mensual.

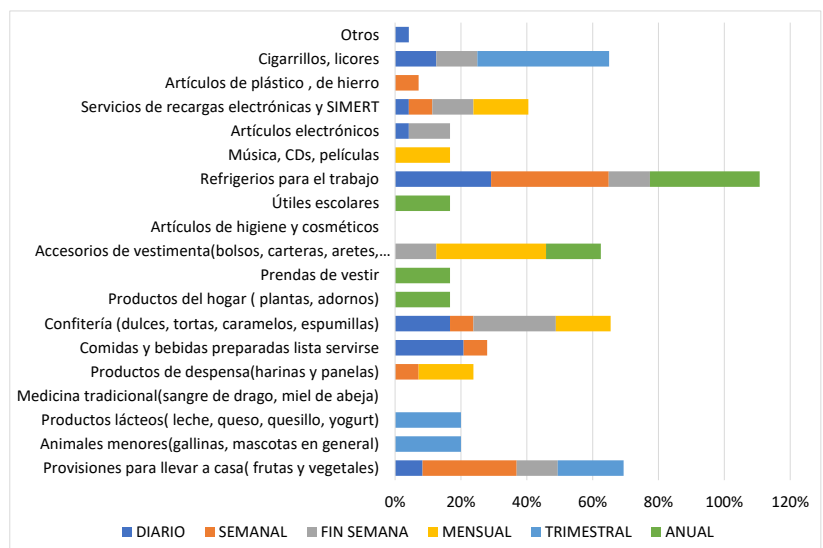

Figura 1. Productos y frecuencia de compra en la movilidad de los consumidores hacia su lugar de trabajo

Fuente: Encuesta

\section{b.- Movilidad hacia su residencia}

Las provisiones llevan a sus residencias tales como frutas y verduras, su frecuencia de compra es en fines de semana y durante la semana; productos lácteos tienen una frecuencia de compra semanal y en fines de semana: comidas y bebidas preparadas listas para servirse estas se adquieren en forma diaria con un $24 \%$ de respuestas favorables; la compra de confitería tiene una frecuencia del $17 \%$ de consumo diario y en porcentajes significativos durante la semana, fines de semana y mensualmente. Las prendas de vestir, animales menores, accesorios de vestimenta, prendas de vestir y música y cds tienen frecuencia de compra mensual, trimestral y anual en porcentajes que van del $10 \%$ como mínimo. (Ver figura 2) 


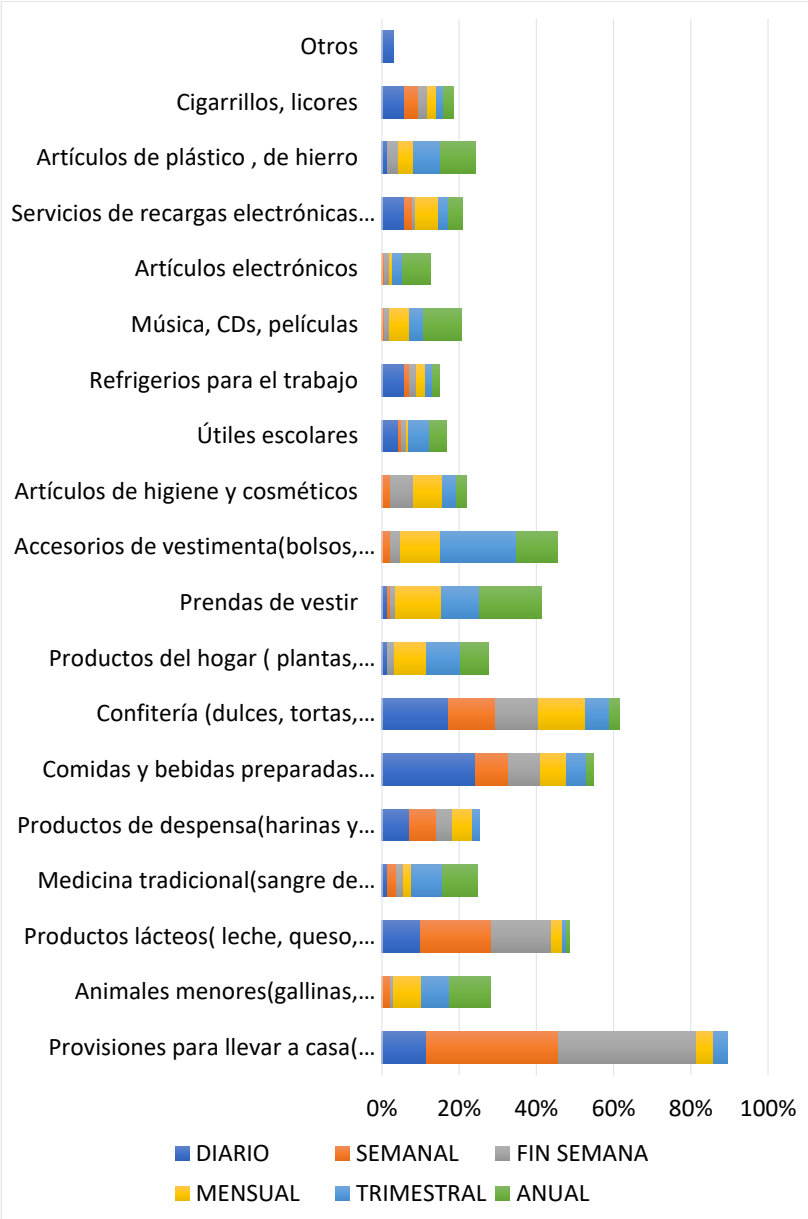

Figura 2. Productos y Frecuencia de Compra en la Movilidad de los Consumidores hacia su residencia

\section{Fuente: Encuesta}

\subsubsection{Motivación de la compra}

\section{a.- Sensibilidad}

Cuando se les indagó porque adquieren el producto un $36 \%$ manifestó porque el vendedor es niño, además porque es pobre contestó un $32 \%$ de entrevistados y porque es mamá en menor porcentaje.

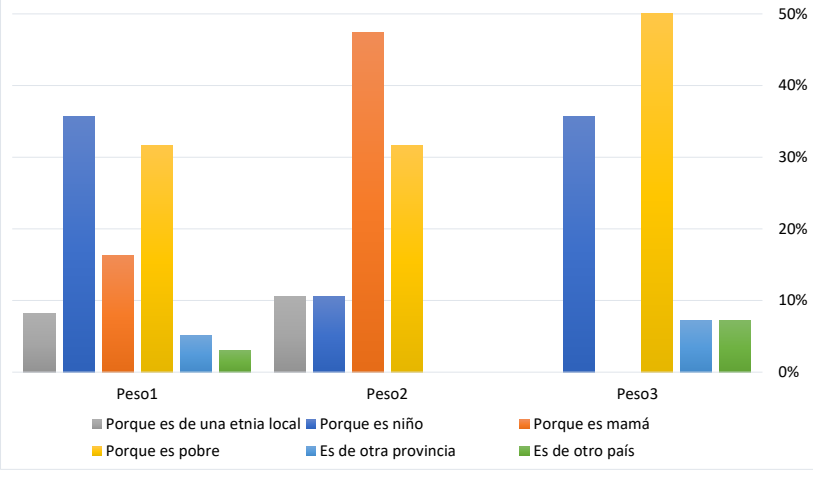

Figura 3. Sensibilidad de Consumidor

Fuente: Encuesta

\section{b.- Mercadeo}

Al evaluar esta característica, un $66 \%$ de los encuestados manifestó que compra porque su precio es cómodo y en menor proporción porque satisface sus expectativas; como opción adicional son el peso adecuado (yapa), que ofrecen un producto fresco/nuevo y compran por la variedad que ofrecen.

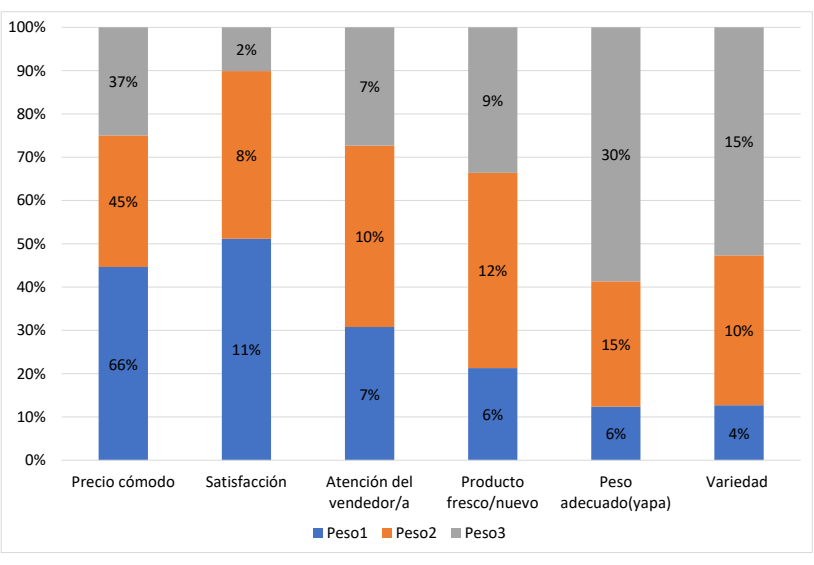

Figura 4. Mercadeo

Fuente: Encuesta

\section{c.- Otros factores}

Cuando se mide otros factores que inciden en la compra, se debe mencionar: el fácil acceso a los vendedores, la calidad de los productos, la urgencia del consumidor y la accesibilidad rápida al vendedor.

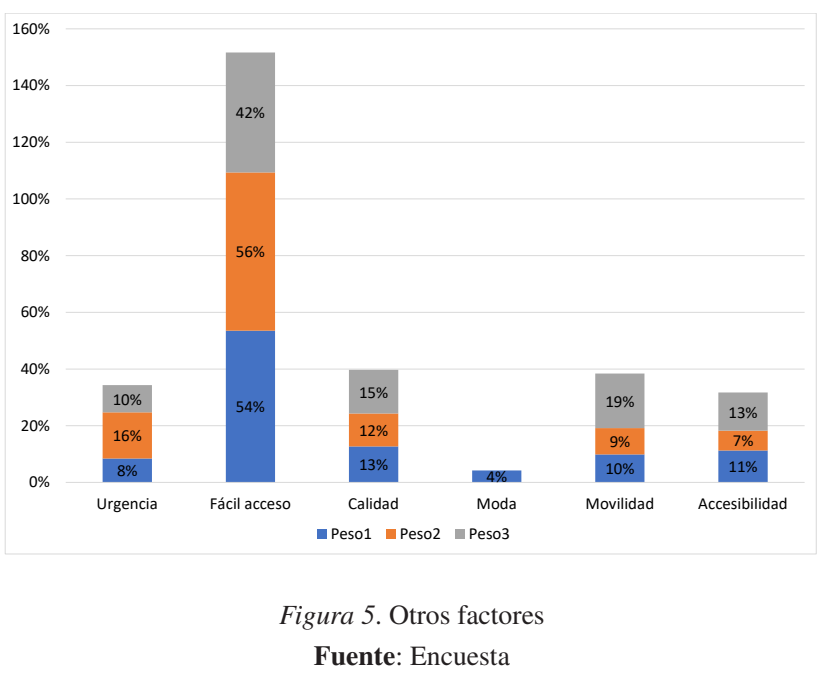

\subsubsection{Lugar habitual de compra}

Los lugares habituales de mayor frecuencia de compra según los datos contrastados son las inmediaciones del mercado Mayorista y Gran Colombia; la calle 10 de agosto; calle 18 de noviembre; y Rocafuerte entre 18 de Noviembre y Lauro Guerrero. Puesto que aquí se encuentran las paradas de los buses desde el centro de la ciudad hacia los barrios urbano marginales y a la vez son la llegada de los 
consumidores desde los barrios hasta el centro de la ciudad de Loja. Asi lo demuestra la figura 6

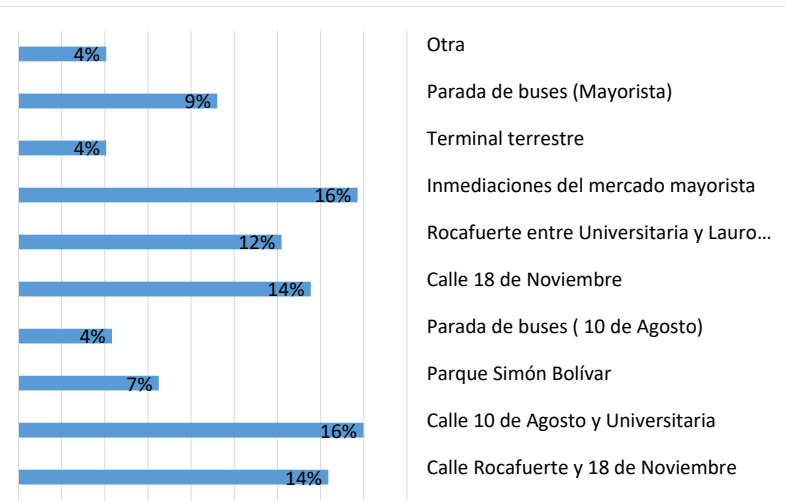

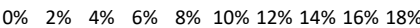

Figura 6. Lugar donde Adquiere Productos a los Vendedores Ambulantes Fuente: Encuesta

\section{Conclusiones}

1) Las características socioeconómicas de los consumidores ambulantes son aquellos que están comprendidos entre los 19 y 50 años de edad, no hay diferencia en el consumo respecto al género, son de estado civil solteros y casados; las ocupaciones principales de ellos son estudiantes, empleados públicos y empleados privados, profesores y amas de casa: con estudios de educación superior y en menor proporción han estudiado el bachillerato; y provienen del cantón Loja en su mayoría, de los barrios y parroquias especialmente El Valle y Sucre en su gran mayoría, puesto que los barrios que las conforman a estas, se encuentran distantes de los centros de consumo y acuden a estos por tema de trabajo y gestiones en instituciones públicas y privadas.

2) Cuando se habla de la frecuencia de compra a los vendedores ambulantes y el tema de movilidad del consumidor de ventas ambulantes, estas mostraron que cuando estos acuden a sus lugares de trabajo diariamente estos consumen comidas y bebidas preparadas listas para servirse y llevan refrigerios para el trabajo; y en menor proporción confitería; y cigarrillos y licores. La frecuencia de compra por semana muestra que los consumidores adquieren provisiones para llevar a casa como son frutas y vegetales y refrigerios para el trabajo; los fines de semana se adquiere confitería (dulces, tortas, caramelos, espumillas) y servicios de recargas electrónicas y SIMERT y artículos electrónicos, accesorios de vestimenta. Mensualmente adquieren productos de despensa, confitería, accesorios de vestimenta, música y recargas en mayor proporción. Y en menor frecuencia de compra se realiza trimestral y anualmente se adquieren productos como provisiones para llevar a casa, animales menores, productos lácteos, cigarrillo y licores, productos del hogar, prendas de vestir, entre otros.
3) Cuando se consultó su frecuencia de compra cuando vuelve a casa que tipo de productos adquiere se encontró comidas y bebidas preparadas, provisiones, confitería y productos lácteos en forma diaria; semanalmente se adquiere provisiones, productos lácteos, confitería; durante los fines de semana se adquiere provisiones, productos lácteos y confitería. Y en forma mensual, trimestral y anual productos lácteos, confitería, accesorios para vestimenta.

4) Los factores que influyen al momento de comprar, el primer factor a analizar es la sensibilidad, es decir, estos compran porque el vendedor es niño, es pobre, y/o es mamá. Cuando se considera la variable mercadeo se compra porque el precio es cómodo, por la satisfacción que adquiere el consumidor, por el peso adecuado(yapa); y, cuando se mide otros factores que inciden en la compra se menciona: por el fácil acceso, urgencia de compra, calidad del producto y movilidad.

5) cuando se les preguntó en que sitio adquieren el producto, se encontró las inmediaciones del mercado Mayorista y Gran Colombia, la calle 10 de agosto, calle 18 de noviembre y calle Rocafuerte. Esto se asocia con las paradas de los buses y concentración de las instituciones públicas y privadas en el casco céntrico de la ciudad.

\section{Referencias Bibliográficas}

Cardona Madeline, M. (2016). Canal formal e informal: Elecciòn del consumidor. Redmarka Académica de Marketing Aplicado, 27.

Duhau Giglia, E. y. A. (2007). Nuevas centralidades y prácticas de consumo en la Ciudad de México: del microcomercio al hipermercado. EURE, 77-95.

Garces. (1999). Manual de Imformaciòn y Auto ayuda: La adiccion al consumo. Unión de Consumidores de España, 56.

Rocha, R., Sanchez, F., y Garcìa, L. (2009). Ventas callejeras y espacio público:. DESARROLLO Y SOCIEDAD, 245-268.

Recibido: 30 de junio de 2018

Aceptado: 3 de septiembre de 2018 